\title{
LARGE SCALE STRUCTURES OF THE UNIVERSE
}

\author{
JEAN AUDOUZE, MARIE-CHRISTINE PELLETAN \\ and ALEX SZALAY (EDS.)
}

The knowledge of the spatial distribution and the evolution with time of large scale structures (clusters of galaxies, hypothetical superclusters) constitutes one of the most important goals of observational and physical cosmology.

Any cosmological theory should reproduce these distributions, which are quite anisotropic and inhomogeneous. The discoveries of new voids, bubbles, and sponges are described at length in this book. These structures provide invaluable constraints on many important cosmological and physical issues such as the distribution and the nature of the so-called dark matter, the possible existence of cosmic strings, etc.

All the observational and the theoretical aspects of this fast-moving topic have been fully described, analyzed and discussed by the entire international cosmological community.

This book is indeed the most up to date account on our knowledge of the overall structure of the Universe.

\section{KLUWER ACADEMIC PUBLISHERS}

DORDRECHT / BOSTON / LONDON 